\title{
Encapsulamiento de lodos de plantas de tratamiento de aguas residuales de la industria automotriz en matrices de arcilla
}

\section{Encapsulation of sludge from the sewage treatment plants of the clay matrices automotive industry}

\section{César Augusto García Ubaque}

Ingeniero Civil, doctor en Ingeniería. Docente Asistente de la Universidad Distrital Francisco José de Caldas. Bogotá, Colombia.

Contacto: cagarciau@udistrital.edu.co.

María Camila García Vaca

Ingeniera Química. Estudiante de maestría en Ingeniería Química de la Universidad de los Andes. Bogotá, Colombia. Contacto: mc.garcia149@uniandes.edu.co.

\section{Martha Lucía Vaca Bohórquez}

Psicóloga, MBA. Consultora de la Universidad de los Andes. Bogotá, Colombia. Contacto:ml.vaca68@uniandes.edu.co.

Palabras clave: aglomerantes, eliminación de residuos, ensayo de materiales, materiales cerámicos, productos de arcilla.

Key words: binders, waste disposal, materials testing, ceramic materials, clay products.

\section{RESUMEN}

En el presente artículo, se presentan los resultados de un estudio piloto de lixiviación de metales de piezas cerámicas elaboradas con mezclas de arcilla y lodos provenientes de la planta de tratamiento de aguas residuales industriales de la planta de ensamble de G.M. COLMOTORES en Bogotá (Colombia). Los lodos han sido esta- bilizados y solidificados en arcillas usadas para la fabricación de ladrillo, mediante cocción en un horno ladrillero tipo Hoffman. Se utilizaron las siguientes proporciones de mezcla de arcilla y lodo respectivamente: 99:1, 95:5, 90:10, 80:20 y 60:40. Con estas proporciones se fabricaron lotes de ladrillos que fueron calcinados en un rango de temperatura entre 50 y $1100{ }^{\circ} \mathrm{C}$. Tanto la arcilla, como los lodos y los ladrillos fabrica- 


\section{investigación}

dos fueron caracterizados mediante: Difracción de Rayos X (XRD), Fluorescencia de Rayos X (FRX), Termogravimetría(TG), Análisis Térmico Diferencial (DTA), Espectroscopia de Absorción atómica (AA) y Microscopia electrónica de barrido (SEM). Adicionalmente, se realizaron pruebas de lixiviación (Toxicity Characteristic Leaching Proccedure -TCLP) para la determinación de metales pesados. Los resultados encontrados, muestran que existe afinidad entre la arcilla y este tipo de lodos y que los ladrillos fabricados con estas mezclas presentan bajos niveles de lixiviación. Se encuentra que la proporción de mezcla que permite la mayor remoción para todos los metales considerados es 95:5 de arcilla y lodo respectivamente. El metal con menor porcentaje de remoción es el Selenio y los que presentan mayores porcentajes son: Arsénico, Niquel, Cromo, Cinc y Cadmio, con excepción de este último en la mezcla 99:1.

\section{ABSTRACT}

This article presents results of a pilot study, where metals leaching from ceramic pieces were made with different mixes of slugde and clay from the sewage treatment plant G.M. COLMOTORES in Bogota (Colombia). The slugde have been stabilized and solidified in clays and cook in a Hoffman Furnace oven for the fabrication of bricks. The proportions of mixture of clay and sludge for the fabrication of bricks used were: 99:1, 95:5, 90:10, 80:20 and 60:40. Then, the bricks were burned at temperatures between 50 to $1100^{\circ} \mathrm{C}$. Moreover, clays, sludge and bricks were tested by: X ray diffraction (XRD), X-ray Fluorescence $\mathrm{X}$ (XRF), Thermogravimetry (TG), Differential Thermal Analysis (DTA), Atomic Absorption Spectroscopy (AA) and Scanning Electron Microscopy (SEM). Finally, other leaching tests were performed in the bricks (Toxicity Characteristic Leaching Procedure - TCLP) for the determination of heavy metals. Results from this study showed that the clay had a high affinity with the sludge and the bricks showed low levels of leaching. Also, it was considered that 95:5 of clay and sludge was the best mixing ratio for all the metals. The metal with lowest removal percentage was selenium and the highest removal percentages were arsenic, nickel, chrome, zinc and cadmium.

\section{INTRODUCCIÓN}

Los lodos de aguas residuales y de algunos procesos industriales son considerados como residuos peligrosos, que demandan tratamiento y manejo especial desde el punto de vista ambiental y sanitario. Están formados generalmente por sólidos en forma de grumos o escamas coloidales mezclados con agua. La parte sólida de los lodos está compuesta por partículas de materia orgánica e inorgánica que tienen rangos de tamaño entre arenas y limos. La disposición final de este tipo de residuos es un problema ambiental crítico en la mayoría de países latinoamericanos y solamente algunas de las principales ciudades cuentan con métodos de disposición adecuados [1].

Souza Santos [2] define una arcilla, como un material terroso de baja granulometría y que presenta plasticidad cuando es mezclado con cierta cantidad de agua. Las arcillas están constituidas por partículas cristalinas extremadamente pequeñas $(<2 \mu \mathrm{m})$ denominadas "minerales de arcilla", de naturaleza inorgánica, compuesta principalmente por $\mathrm{Si}, \mathrm{Al}, \mathrm{H}_{2} \mathrm{O}$ y, frecuentemente, cantidades apreciables de Fe y metales alcalinos y alcalinotérreos. Además de minerales, una arcilla puede contener materia orgánica y otros compuestos no cristalinos o amorfos.

Los componentes principales de las arcillas usadas en la industria ladrillera son: óxido de sílice, óxido de aluminio y silicatos de aluminio [3]. 
Estos materiales similares a las zeolitas naturales también actúan como intercambiadores iónicos [4]. Por otro lado, los lodos provenientes de la planta de tratamiento de aguas residuales industriales de la empresa GM COLMOTORES, contienen algunos componentes típicos que también se encuentran en las arcillas, como $\mathrm{SiO}_{2}, \mathrm{Al}_{2} \mathrm{O}_{3}$ y $\mathrm{CaO}$ [5] que facilitan su compatibilidad para hacer mezclas con ellas. Esta afinidad, sumada a la capacidad de intercambio iónico, parece ser el principal mecanismo que hace posible el encapsulamiento de este tipo de lodos en las arcillas durante la etapa de cocción dentro del proceso ladrillero, por lo que puede ser considerado como un proceso de Solidificación - Estabilización [6].

Algunos procesos que tratan residuos peligrosos con diferentes materiales como: cemento, arcillas, polímeros y otros, son conocidos como técnicas de "Solidificación-Estabilización". Diferentes autores las han estudiado usando una gran variedad de residuos y matrices, de hecho, la industria de cemento cuenta con una amplia experiencia en la aplicación de estas tecnologías [7-8].

Recientemente, se han usado arcillas marinas para estabilizar residuos del proceso de hidrogenación catalítica, como $\mathrm{Co} / \mathrm{Mo} / \mathrm{Al}_{2} \mathrm{O}_{3}$ y Ni/Mo/ $\mathrm{Al}_{2} \mathrm{O}_{3}$, en la industria petrolera. El material resultante fue usado para fabricar ladrillos mediante procesos de vitrificación [4]. De acuerdo con los resultados de sus pruebas de lixiviación, los autores proponen un mecanismo para explicar el pro- ceso de estabilización, que involucra dos fases: en la primera, la arcilla forma una matriz alrededor de metales y los encapsula; y en la segunda, los metales pesados forman enlaces químicos con la arcilla y por tanto se incorporan a la matriz a temperaturas mayores a $1000^{\circ} \mathrm{C}$.

\section{MATERIALES Y MÉTODOS}

Para determinar las características de los lodos utilizados en este estudio, se llevaron a cabo los siguientes análisis de laboratorio, en una muestra puntual de $500 \mathrm{~g}$ :

- Termogravimetría

- Espectroscopía Infraroja

- Difracción de Rayos X

- Análisis de metales por Absorción Atómica

Para la caracterización de las arcillas del proceso, fueron recolectadas muestras de $100 \mathrm{~g}$, cada hora durante 30 días de operación de la planta. Cuando se completaron $10 \mathrm{~kg}$, se seleccionaron tres muestras y su composición elemental fue determinada usando técnicas de XRF y XRD, DTA, AAS, SEM. Los análisis de laboratorio se llevaron a cabo en los laboratorios de Sólidos Porosos y Microcalorimetría del departamento de Química de la Universidad de los Andes en Bogotá (Colombia) y en el laboratorio de Ciencias de Superficies

Tabla 5. Equipos empleados en las diferentes determinaciones

\begin{tabular}{|l|l|l|l|}
\hline ANÁLISIS & EQUIPO & MODELO & CARACTERÍSTICAS \\
\hline DRX & RIGAKU & MINIFLEX & $2-70^{\circ}\left(\mathrm{c} / 0.02^{\circ}\right), 2^{\circ} / \mathrm{min}$ \\
\hline FRX & PHILLIPS & PW 1400 & Tubo RX, Ánodo de Rh, Colimador HR. \\
\hline ATD & NETZSCH & STA 409 PC LUX & $30-1100^{\circ} \mathrm{C}, 15^{\circ} / \mathrm{min}$ \\
\hline AA & PERKIN ELMER & AANALYST 300 & \\
\hline
\end{tabular}

Fuente: elaboración propia 


\section{investigación}

y Medios Porosos de la Universidad Nacional de San Luis en San Luis (Argentina). Los equipos utiliza dos se describen en la tabla 1.

Además de las muestras recolectadas, otras tres muestras fueron seleccionadas aleatoriamente en diferentes días y a diferentes horas. Estas muestras se analizaron de la misma manera para validar los resultados obtenidos.

El lodo y la arcilla se usaron para preparar cinco mezclas -húmedas- con las siguientes proporciones en peso de arcilla/lodo: 100:0, 99:1, 95:5, 90:10, 80:20 y 60:40. Cada mezcla fue usada para preparar 10 ladrillos de prueba de $200 \mathrm{~g}$ cada uno. Estos ladrillos se calcinaron en laboratorio, utilizando un horno marca BARNSTESD modelo 48000, siguiendo de manera aproximada la curva típica de temperatura del horno Hoffman. En la figura 1 se muestra el comportamiento de la temperatura que fue programado en los controladores del horno empleado para la cocción de los ladrillos.
Después de calcinados los ladrillos de prueba, se seleccionaron, de manera aleatoria, tres unidades de cada lote, para realizar pruebas y determinar la proporción óptima de arcilla-lodos. Las piezas seleccionadas fueron caracterizadas usando las mismas técnicas aplicadas inicialmente con la arcilla y los lodos. Finalmente, se les aplicó pruebas de lixiviación (TCLP), ya que este ensayo está diseñado para determinar si un analito orgánico o inorgánico, presente en un desecho sólido o líquido, se moviliza al lixiviado por acción de un medio fuertemente ácido. La prueba consiste en la extracción sólido-líquido de la mezcla del material previamente molido y tamizado hasta un tamaño de partícula menor de 20 $\mu \mathrm{m}$ con agua regia -mezcla de ácido clorhídrico y ácido nítrico- durante varias horas, permitiendo el contacto directo del material con el medio ácido. Luego de la extracción, se realizan diluciones específicas del extracto para el análisis de cada uno de los componentes metálicos por AA. Los resultados de los ensayos de lixiviación fueron comparados con la concentración inicial de metales pesados en los lodos. La disminución en

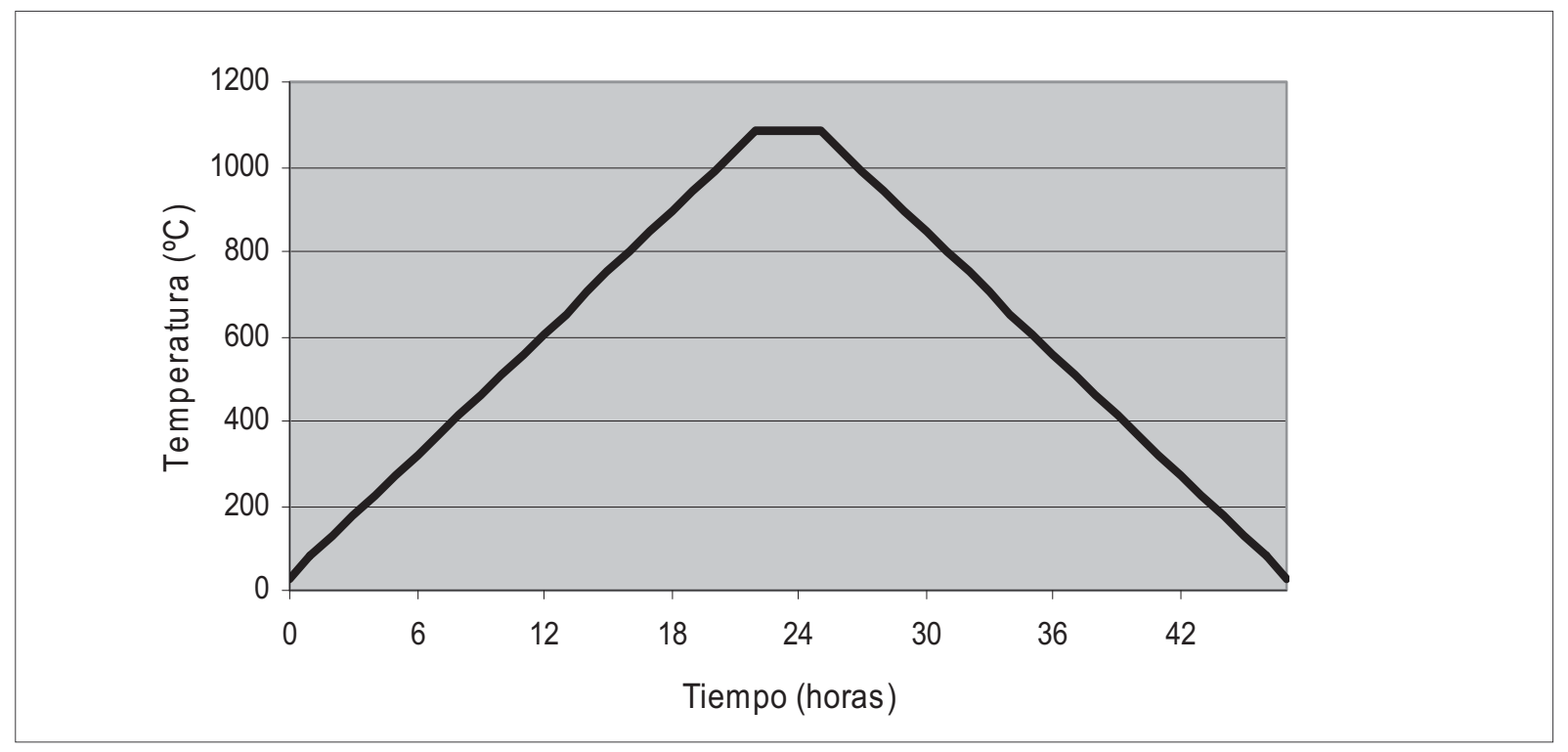

Figura 1. Curva de cocción en laboratorio

Fuente: elaboración propia. 
la concentración de metal fue calculada como se expresa en la ecuación (1).

$$
\% \text { Encapsulamiento }=\frac{C_{0}-C_{T C L P}}{C_{0}} * 100
$$

Donde:

$$
\begin{aligned}
\mathrm{C}_{\mathrm{o}}= & \begin{array}{l}
\text { Concentración inicial del metal } \\
\text { en los lodos. }
\end{array} \\
\mathrm{C}_{\mathrm{TCLP}}= & \begin{array}{l}
\text { Resultado obtenido en la prueba } \\
\text { de lixiviación }
\end{array}
\end{aligned}
$$

\section{RESULTADOS Y DISCUSIÓN}

\subsection{Caracterización de los lodos de PTAR}

En las tablas 2 y 3 se presentan los resultados del análisis físico químico y de metales pesados de los lodos provenientes de la PTAR.

El contenido de humedad en los lodos utilizados es similar al de la arcilla, por lo que no es necesario realizar ningún tipo de tratamiento previo al encapsulamiento dado que la formación de los ladrillos se realiza en condiciones de humedad cercanas al $30 \%$.

El análisis de metales de las muestras de lodos reporta concentraciones por debajo de los niveles máximos permitidos para todos los metales estudiados en los ensayos de lixiviación. Estos resultados se ilustran en la tabla 3.

Tabla 6. Análisis físico químico de los lodos utilizados

\begin{tabular}{|l|c|}
\hline Parámetro & Valor (\%) \\
\hline $\mathrm{PH}$ & 6,5 \\
\hline Humedad & 16,2 \\
\hline Materia Orgánica (Sólidos Volátiles) & 42,3 \\
\hline $\begin{array}{l}\text { Material Inorgánico }\left(\mathrm{SiO}_{2}, \mathrm{Al}_{2} \mathrm{O}_{3}, \mathrm{CaO},\right. \\
\left.\mathrm{Fe}_{2} \mathrm{O}_{3}\right)\end{array}$ & 35,0 \\
\hline
\end{tabular}

Fuente: elaboración propia
Tabla 7. Concentración de metales pesados en los lodos utilizados

\begin{tabular}{|l|c|c|}
\hline Metal & Concentración & $\begin{array}{c}\text { Nivel máximo } \\
\text { permitido } \\
\text { (mg/kg) }\end{array}$ \\
\hline Arsénico (As) & 1,12 & 5,0 \\
\hline Bario (Ba) & 2,26 & 100,0 \\
\hline Cadmio (Cd) & 0,43 & 1,0 \\
\hline Cromo Total (Cr) & 3,45 & 5,0 \\
\hline Cobre (Cu) & 1,56 & - \\
\hline Mercurio (Hg) & 0,023 & 0,2 \\
\hline Magnesio (Mg) & 4,72 & - \\
\hline Níquel (Ni) & 3,37 & - \\
\hline Plomo (Pb) & 1,23 & 5,0 \\
\hline Plata (Ag) & 0,083 & 5,0 \\
\hline Selenio (Se) & 0,221 & 1,0 \\
\hline Zinc (Zn) & 2,27 & - \\
\hline
\end{tabular}

Fuente: elaboración propia

\subsection{Caracterización de la arcilla del proceso}

La figura 2 muestra el difractograma obtenido en el análisis de Difracción de Rayos X de las arcillas colombianas. Caolinita, Illita, Montmori1lonita y Cuarzo son los principales compuestos identificados.

\subsection{Resultados de las ensayos de lixiviación en los ladrillos de prueba}

La tabla 4 resume los resultados de las pruebas de lixiviación según la proporción de arcilla-lodos usada. La figura 3 ilustra estos resultados. El porcentaje de encapsulamiento fue calculado según la ecuación (1).

De forma aproximada, el porcentaje de disminución en la concentración de metales puede interpretarse como el porcentaje de encapsulamiento; dado que por las condiciones extremas del ensa- 


\section{investigación}

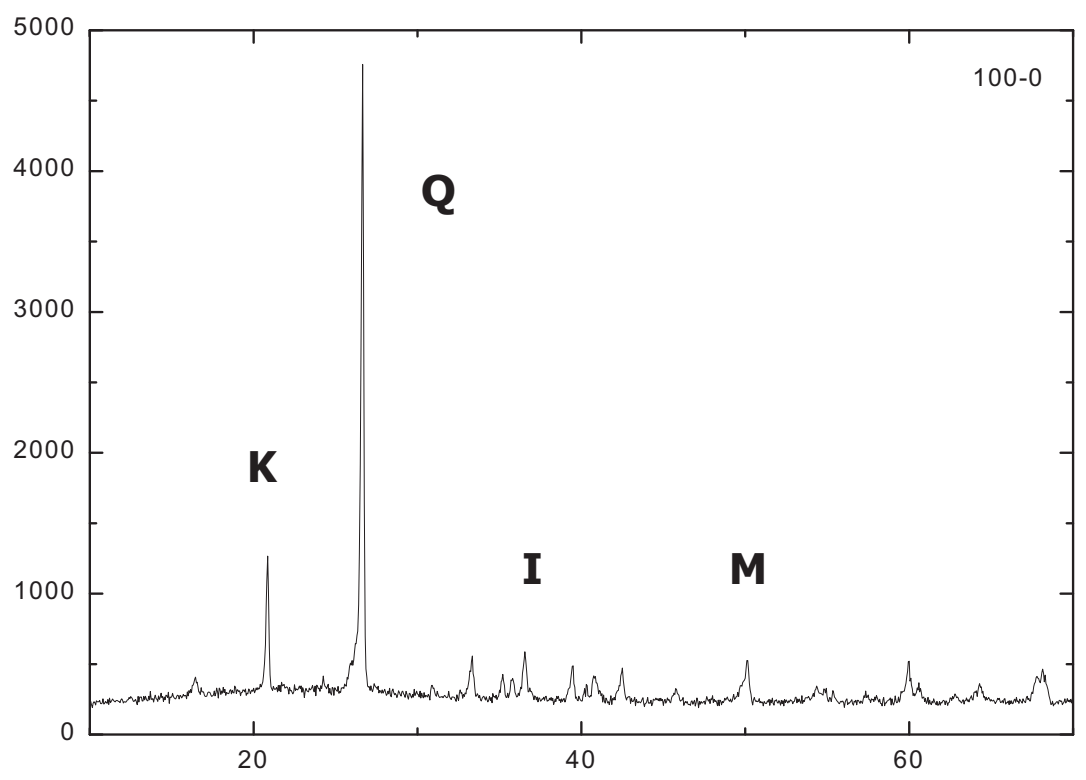

Figura 2. Difractograma de las arcillas utilizadas

Fuente: elaboración propia.

Tabla 8. Contenido de metales pesados en ladrillos fabricados con las mezclas de arcilla y lodos

\begin{tabular}{|c|c|c|c|c|c|c|}
\hline$\%$ Arcilla-Lodos & & $99-1$ & $95-5$ & $90-10$ & $80-20$ & $60-40$ \\
\hline \multicolumn{7}{|c|}{ Arsénico (As) } \\
\hline Resultado del TCLP & \multirow{2}{*}{$\mathrm{mg} / \mathrm{kg}$} & 0,001 & 0,0012 & 0,002 & 0,0076 & 0,0124 \\
\hline Concentración inicial & & 1,12 & 1,12 & 1,12 & 1,12 & 1,12 \\
\hline \% Encapsulamiento & $\%$ & 99,91 & 99,89 & 99,82 & 99,32 & 98,89 \\
\hline \multicolumn{7}{|c|}{ Bario (Ba) } \\
\hline Resultado del TCLP & \multirow{2}{*}{$\mathrm{mg} / \mathrm{kg}$} & 0,451 & 0,474 & 0,652 & 0,745 & 0,9425 \\
\hline Concentración inicial & & 2,26 & 2,26 & 2,26 & 2,26 & 2,26 \\
\hline$\%$ Encapsulamiento & $\%$ & 80,04 & 79,03 & 71,15 & 67,04 & 58,3 \\
\hline \multicolumn{7}{|c|}{ Cadmio (Cd) } \\
\hline Resultado del TCLP & \multirow{2}{*}{$\mathrm{mg} / \mathrm{kg}$} & 0,2 & 0,0287 & 0,0314 & 0,0354 & 0,0568 \\
\hline Concentración inicial & & 0,43 & 0,43 & 0,43 & 0,43 & 0,43 \\
\hline \% Encapsulamiento & $\%$ & 53,49 & 93,33 & 92,7 & 91,77 & 86,79 \\
\hline \multicolumn{7}{|c|}{ Cromo total $(\mathrm{Cr})$} \\
\hline Resultado del TCLP & \multirow{2}{*}{$\mathrm{mg} / \mathrm{kg}$} & 0,0356 & 0,0479 & 0,0502 & 0,0738 & 0,104 \\
\hline Concentración inicial & & 3,45 & 3,45 & 3,45 & 3,45 & 3,45 \\
\hline \% Encapsulamiento & $\%$ & 98,97 & 98,61 & 98,54 & 97,86 & 96,99 \\
\hline
\end{tabular}




\section{investigación}

\begin{tabular}{|c|c|c|c|c|c|c|}
\hline$\%$ Arcilla-Lodos & & $99-1$ & $95-5$ & $90-10$ & $80-20$ & $60-40$ \\
\hline \multicolumn{7}{|c|}{ Mercurio (Hg) } \\
\hline Resultado del TCLP & \multirow{2}{*}{$\mathrm{mg} / \mathrm{kg}$} & ND & ND & ND & ND & ND \\
\hline Concentración inicial & & 0,023 & 0,023 & 0,023 & 0,023 & 0,023 \\
\hline \% Encapsulamiento & $\%$ & - & - & - & - & - \\
\hline \multicolumn{7}{|c|}{ Níquel (Ni) } \\
\hline Resultado del TCLP & \multirow{2}{*}{$\mathrm{mg} / \mathrm{kg}$} & 0,027 & 0,038 & 0,041 & 0,052 & 0,067 \\
\hline Concentración inicial & & 3,37 & 3,37 & 3,37 & 3,37 & 3,37 \\
\hline \% Encapsulamiento & $\%$ & 99,2 & 98,87 & 98,78 & 98,46 & 98,01 \\
\hline \multicolumn{7}{|c|}{ Plata (Ag) } \\
\hline Resultado del TCLP & \multirow{2}{*}{$\mathrm{mg} / \mathrm{kg}$} & 0,03 & 0,0321 & 0,0345 & 0,03 & 0,034 \\
\hline Concentración inicial & & 0,083 & 0,083 & 0,083 & 0,083 & 0,083 \\
\hline \% Encapsulamiento & $\%$ & 63,86 & 61,33 & 58,43 & 63,86 & 59,04 \\
\hline \multicolumn{7}{|c|}{ Plomo (Pb) } \\
\hline Resultado del TCLP & \multirow{2}{*}{$\mathrm{mg} / \mathrm{kg}$} & 0,2 & 0,0236 & 0,427 & 0,427 & 0,521 \\
\hline Concentración inicial & & 1,23 & 1,23 & 1,23 & 1,23 & 1,23 \\
\hline \% Encapsulamiento & $\%$ & 83,74 & 98,08 & 65,28 & 65,28 & 57,64 \\
\hline \multicolumn{7}{|c|}{ Selenio (Se) } \\
\hline Resultado del TCLP & \multirow{2}{*}{$\mathrm{mg} / \mathrm{kg}$} & 0,0145 & 0,0132 & 0,0126 & 0,0126 & 0,0136 \\
\hline Concentración inicial & & 0,021 & 0,021 & 0,021 & 0,021 & 0,021 \\
\hline \% Encapsulamiento & $\%$ & 30,95 & 37,14 & 40,00 & 40,00 & 35,24 \\
\hline \multicolumn{7}{|l|}{$\operatorname{Zinc}(Z n)$} \\
\hline Resultado del TCLP & \multirow{2}{*}{$\mathrm{mg} / \mathrm{kg}$} & 0,023 & 0,0326 & 0,0387 & 0,0723 & 0,104 \\
\hline Concentración inicial & & 2,27 & 2,27 & 2,27 & 2,27 & 2,27 \\
\hline \% Encapsulamiento & $\%$ & 98,99 & 98,56 & 98,3 & 96,81 & 95,42 \\
\hline
\end{tabular}

Fuente: elaboración propia

32 Tecnura | Vol. $17 \mid$ No. 38 | Octubre - Diciembre de 2013 


\section{investigación}

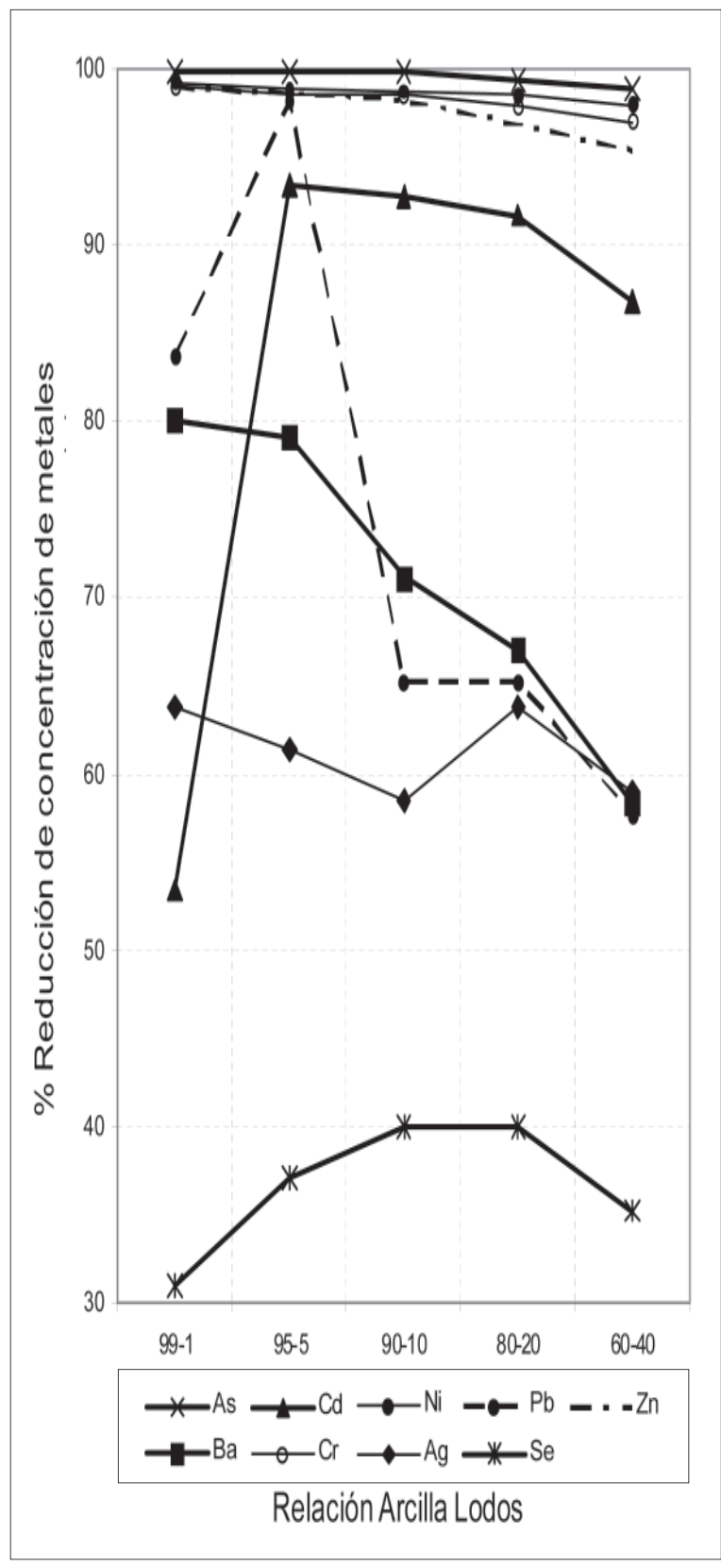

Figura 2. Reducción del contenido de metales Fuente: elaboración propia.

yo, se infiere que las partículas metálicas que no lixivian, permanecen en alguna forma estables al interior de la matriz de arcilla.

De manera general, el porcentaje de encapsulamiento decae levemente para Arsénico, Cinc,
Cromo y Níquel, conforme aumenta el contenido de lodos en las mezclas. El Bario también presenta esta tendencia pero el decaimiento es mayor. El hecho de que el decaimiento sea leve para estos metales, puede indicar que no existe un efecto significativo de la proporción de lodos. Este aspecto puede ser es de gran interés a la hora de analizar la cantidad de estos metales que podrían llegar a encapsularse. En concordancia con lo anterior, se puede decir que, para el Bario, el porcentaje de encapsulamiento sí depende significativamente de la proporción de lodos empleada.

Con el Plomo y el Cadmio, la mayor disminución del porcentaje de encapsulamiento con respecto de la proporción de lodos se presenta entre las relaciones 95:5 y 90:10. Se puede deducir que aumentar de $1 \%$ a $5 \%$ la proporción de lodo en la arcilla, es adecuado para el caso del plomo y del cadmio, ya que permite aumentar significativamente la cantidad encapsulada de estos metales y principalmente el cadmio. Proporciones mayores no tienen un efecto significativo en la disminución de cadmio pero sí lo tienen en la disminución del encapsulamiento del plomo. Entre las proporciones $5 \%$ y $10 \%$ de lodo la disminución del encapsulamiento se encuentra entre $98,08 \%$ y $65,28 \%$.

En el caso de la plata, el porcentaje de encapsulamiento disminuye en las primeras tres proporciones (99:1, 95:5 y 90:10), pero se presenta un aumento en la proporción 80:20 y luego disminuye nuevamente. La tendencia para el selenio también presenta un comportamiento diferente. Inicialmente, se observa un aumento progresivo del porcentaje de encapsulamiento entre la proporción 99:1 hasta la proporción 80:20, pero luego, el porcentaje de encapsulamiento decae, lo que permite afirmar que, para este metal, es conveniente trabajar con proporciones de lodos del $20 \%$, ya que es el que permite alcanzar el mayor porcentaje de encapsulamiento. 


\section{investigación}

Tabla 9. Porcentajes de metal no removido para diferentes concentracionesde lodo

\begin{tabular}{|c|c|c|c|c|c|}
\hline \multirow{2}{*}{ Metal } & \multicolumn{5}{|c|}{ Porcentaje en peso de lodos } \\
\cline { 2 - 6 } & $\mathbf{1} \%$ & $\mathbf{5 \%}$ & $\mathbf{1 0} \%$ & $\mathbf{2 0} \%$ & $\mathbf{4 0} \%$ \\
\hline $\mathrm{As}$ & 0,0 & 0,02 & 0,09 & 0,59 & 1,02 \\
\hline $\mathrm{Ba}$ & 0,0 & 1,01 & 8,89 & 13,0 & 21,74 \\
\hline $\mathrm{Cd}$ & 39,84 & 0,0 & 0,63 & 1,56 & 1,11 \\
\hline $\mathrm{Cr}$ & 0,0 & 0,36 & 0,43 & 0,74 & 1,98 \\
\hline $\mathrm{Ni}$ & 0,0 & 0,33 & 0,42 & 0,0 & 4,19 \\
\hline $\mathrm{Ag}$ & 0,0 & 2,53 & 5,43 & 32,8 & 4,82 \\
\hline $\mathrm{Pb}$ & 14,34 & 0,0 & 32,8 & 0,0 & 40,44 \\
\hline $\mathrm{Se}$ & 9,05 & 2,86 & 0,0 & 2,18 & 4,76 \\
\hline $\mathrm{Zn}$ & 0,0 & 0,43 & 0,69 & & 3,57 \\
\hline
\end{tabular}

Fuente: elaboración propia

El análisis de la disminución en la concentración de mercurio no puede realizarse puesto que su concentración, una vez realizado el proceso, está por debajo del mínimo detectado por el equipo, lo que puede deberse a su alta volatilidad.

Con el objetivo de determinar cuál es la proporción de arcilla-lodo más conveniente se toman en cuenta las siguientes consideraciones: a) la proporción de lodos debe ser tan alta como sea posible con el fin de utilizar la mayor cantidad de lodos; y b) dicha proporción debe maximizar la cantidad de metales encapsulada garantizando que los valores de concentración en el lixiviado sean menores a los que establece la normatividad internacional.

Para todos los metales estudiados se tiene que los mayores porcentajes de encapsulamiento se presentan en los ladrillos producidos con proporciones de lodo entre el 1 y el $5 \%$ con excepción del selenio. En la tabla 5, para cada metal se presenta una cuantificación de la magnitud que dejaría de encapsularse al emplear diferentes proporciones de lodos, con respecto a la proporción en la cual se encapsula la mayor cantidad de cada uno.
Con una proporción del $1 \%$, se trata una menor cantidad de lodos. Adicionalmente, el encapsulamiento de $\mathrm{Se}, \mathrm{Cd}$ y $\mathrm{Pb}$ serían menores en un $9,05 \%, 39,84 \%$, y $14,34 \%$ respectivamente, que si se usaran las proporciones en donde la remoción de estos metales es máxima (10\% y $20 \%)$. Sin embargo, para los demás metales (As, $\mathrm{Ba}, \mathrm{Cr}$, $\mathrm{Ni}, \mathrm{Ag}, \mathrm{y} \mathrm{Zn}$ ), esta proporción permite el mayor nivel de encapsulamiento. Con un porcentaje de lodo de $10 \%$, se deja de remover $8,89 \%$ de $\mathrm{Ba}$, $5,43 \%$ de Ag y $32,8 \%$ de plomo, pero el encapsulamiento de Se es máximo. Los demás metales no se inertizan al máximo, pero los porcentajes de no encapsulamiento respecto del máximo son menores al $1 \%$. Al usar la proporción de $20 \%$ de lodos, se incrementa la fracción no encapsulada de Ba al 13\% y también se observan aumentos de esta fracción en los demás metales. El encapsulamiento de $\mathrm{Pb}$ permanece constante con respecto a la proporción del $10 \%$ de lodos, mientras que para Ag y Se es máximo.

Al $40 \%$ de lodos se presenta un máximo de no encapsulamiento para $\mathrm{As}, \mathrm{Ba}, \mathrm{Cr}, \mathrm{Ni}, \mathrm{Zn}$ y $\mathrm{Pb}$ que asciende al 40,44\%, todos los porcentajes de no encapsulamiento ascienden de manera significa- 


\section{investigación}

tiva. Esto significa que aunque la proporción de lodos es la mayor, el porcentaje de metales no encapsulados es muy elevado, sobre todo en el caso del $\mathrm{Pb}$ y del $\mathrm{Ba}$.

\section{CONCLUSIONES}

Aunque la concentración de metales pesados en los lodos sin tratar se encuentra por debajo de los límites aceptados, los resultados de las pruebas lixiviación muestran reducciones significativas de estas concentraciones en los ladrillos de prueba. Los resultados de los ensayos de lixiviación evidencian que la concentración de metales en los ladrillos fabricados con mezclas de lodo y arcilla, son $30 \%$ a $99 \%$ menores que en los lodos en su estado original.

La concentración que permite el mayor encapsulamiento, teniendo en cuenta todos los metales corresponde a $5 \%$ de lodo.

A nivel general, se presenta un alto grado de encapsulamiento de los siguientes metales: Arsénico, Níquel, Cromo, Cinc y Cadmio. El metal que presenta menor encapsulamiento y remoción es el Selenio.

Dado que en los lodos utilizados se hallan de manera simultánea concentraciones de los diferentes metales de interés ambiental, es posible que se produzca competencia en el proceso de estabilización química entre unos y otros iones metálicos, lo que puede explicar la mayor o menor afinidad de las arcillas del proceso por uno u otro metal. Esta afinidad también puede estar dada por el tipo de óxido metálico presente en los lodos y en la arcilla utilizada.

Adicionalmente, y en la medida en que aumenta la temperatura durante el proceso de cocción de ladrillos, se puede dar lugar a cambios en los estados de oxidación de cada mineral, con lo cual se pueden producir cambios en la capacidad de intercambio catiónico.

La composición mayoritaria de caolinita en la arcilla utilizada y la presencia de óxidos metálicos en los lodos, permite suponer que entre los posibles mecanismos de estabilización o encapsulamiento de los metales que pueden ocurrir, se encuentran:

a) Sustitución catiónica en la estructura de la matriz arcillosa, dada por la pérdida de humedad o de los enlaces $\mathrm{H}+$ y $\mathrm{OH}-$ de algunos minerales presentes en la arcilla y en los lodos.

b) Formación de fases muy estables químicamente, como mullita, cristobalita y espinela, a medida que se alcanza la temperatura de cocción de los ladrillos, dado que estas alcanzan los $1100^{\circ} \mathrm{C}$. [9]. La espinela es conocida como una fase cristalina muy estable e inerte químicamente que puede llegar a agrupar una buena cantidad de óxidos metálicos dentro de la estructura de los aluminosilicatos que la conforman [10].

c) Otro posible mecanismo de estabilización que se puede llegar a presentar en las mezclas de arcilla-lodo durante el proceso de cocción de ladrillos es la formación de fases vítreas, por reacciones de oxidación-reducción, pérdida de humedad e intercambio catiónico a las temperaturas de cocción de los ladrillos por encima de los $1000{ }^{\circ} \mathrm{C}$ [9].

Para futuros trabajos, y con el fin de precisar estos mecanismos de estabilización química y la posible selectividad hacia determinados iones metálicos, se sugiere realizar ensayos selectivos de encapsulamiento con lodos que contengan un solo metal o un grupo más reducido de metales susceptibles de lixiviar.

Es recomendable también hacer ensayos de estabilización con mezclas de metales puros, con el 
fin de determinar la mayor afinidad por unos u otros en relación con la composición de la arcilla utilizada.

\section{FINANCIAMIENTO}

Para el desarrollo de este proyecto se contó con el apoyo económico de la empresa INAMCO y el auspicio de COLCIENCIAS.

\section{AGRADECIMIENTOS}

Los autores expresan su agradecimiento a las empresas INAMCO y Ladrillos Los Quiroga, por el apoyo logístico y la colaboración prestada en el desarrollo de los ensayos a escala real, sin lo cual no se habría podido desarrollar este proyecto.

\section{REFERENCIAS}

[1] M. La Grega, P. Buckingham and J. Evans, Hazardous Waste Management, Ed McGraw Hill Inc., 1994.

[2] P. Souza Santos, Tecnología de Argilas, Ed. Universidad de Sao Paulo, 1975.

[3] J. Dijkstra, H. Van Der Slootand and R. Comans, "Process identification and model development of contaminant transport in MSWI bottom ash", Waste Management, Vol. 22, pp. 531-541, 2002

[4] D. Sun, L. Chang, J. Tay, J. Navratil, and C. Easton, "Recovery And Marine Clay Stabilization Of Heavy Metals Present In Spent Hydrotreating Catalysts", Journal of Environmental Engineering, Vol. 127, No. 10, pp. 916-921,2001.

[5] A. García, E. Álvarez and O. Jiménez de Blas, "Sorption of Heavy Metals from Industrial Waste Water by low-cost mineral silicates", Clay Minerals, Vol. 34, pp. 469477, 1999.

[6] S. Arvelakis and F. Frandsen, "Study on Analysis and Characterization Method for
Ash Materials from Incineration Plants", Fuel, Vol. 84, pp. 1725-1738, 2005.

[7] J. Magalhaes, J. Silva, F. Castro and J. Labrincha, "Kinetic study of the immobilization of galvanic sludge in clay-based Matrix", Journal of Hazardous Materials, Vol. 121, pp. 69-78, 2005.

[8] V. Albino, R. Cioffi, M. Pansini and C. Colella,"Disposal of lead containing zeolite sludge in cement matrix", Environmental Technology, Vol. 16, No. 2, pp. 147156, 1995.

[9] L. Xu, W. Guo, T. Wang and N. Yang, "Study on fired bricks with replacing clay by fly ash in high volume ratio", Construction and Building Materials, Vol. 19, No. 3, pp. 243-247, Abr., 2005.

[10] K. Okada, N. Otsuka and J. Ossaka, "Characterization of Spinel Phase Formed in the Kaolin-Mullite Thermal Sequence", Journal of the American Ceramic Society, Vol. 69, No. 10, pp. C-251-C-253, Oct.1986. 